\title{
Measuring Just Noticeable Differences For Haptic Force Feedback: Implications for Rehabilitation
}

\author{
Sonya Allin \\ Human Computer Interaction Institute \\ Carnegie Mellon University \\ sonyaa@cs.cmu.edu
}

\author{
Yoky Matsuoka \\ Robotics Institute \\ Carnegie Mellon University \\ yoky@cs.cmu.edu
}

\author{
Roberta Klatzky \\ Psychology Department \\ Carnegie Mellon University \\ klatzky@cmu.edu
}

\begin{abstract}
This paper describes preliminary work in the use of a virtual environment to derive just noticeable differences (JNDs) for force. Specifically, we look for thresholds of force sensitivity so that we may ultimately construct therapeutic force feedback distortions that stay below these thresholds. Initially, we have concentrated on $J N D$ s as they are applied to the index finger; preliminary data in healthy individuals shows an average JND of approximately $10 \%$. More significantly, the data indicate that visual feedback distortions in a virtual environment can be created to encourage increased force productions by up to 10\%, and that this can be done without a patient's awareness.
\end{abstract}

\section{Introduction}

This paper describes a preliminary effort to evaluate tactile and kinesthetic force perception using a paradigm designed to guide future work in stroke therapy.

Specifically, we derive just noticeable differences (JNDs) for force using a force-feedback device, so that we may use these JNDs to develop therapeutic feedback distortions in a virtual environment. It is our intention to contrive feedback distortions to encourage incremental, yet imperceptible augmentations in force production without a patient's awareness.

The JND is a measure of the minimum difference between two stimuli that is necessary in order for the difference to be reliably perceived. With respect to force, the measure corresponds to the degree of augmentation that an individual can experience before he or she is aware that the augmentation has taken place. In the therapeutic setting, JNDs may prove to be a powerful tool with which to induce gradual and imperceptible increases in a patient's production of force. These increases can be used to subvert a patient's self-imposed limits on force production (such limits are oftentimes referred to as 'Learned Nonuse' [10]). Our ultimate goal, then, is to create a distorted virtual environment that adheres to whatever sense of capability a patient may report, but that extends limitations by perceptually undetectable amounts.

Much prior research has focused on just noticeable differences for force in human subjects but none, to our knowledge, has tailored this knowledge to the rehabilitation domain $[1,3,4,7,8,9]$. In the early part of the 19th century, Weber $(1834 / 1978)$ measured JNDs of roughly $10 \%$ in experiments involving active lifting of 32 oz. weights by the hand and arm [8]. More recent work by Brodie and Ross (1984) has yielded similar JNDs for tasks involving the active lifting of $2 \mathrm{oz}$. weights [1]. Jones (1989), in a force matching experiment about the elbow, found a JND ranging between 5\% and 9\% [3]. Pang, Tan and Durlach (1991) report a JND that lies between 5\% and $10 \%$ for pinching motions between finger and thumb with a constant resisting force [7]. This JND was found to be relatively constant over a range of different base force values between 2.5 and 10 Newtons.

The reality of stroke, however, complicates the questions inherent in this kind of research. JNDs in stroke victims may be different from those found in healthy individuals and other patients, and they may vary between patients with the same disorders. Moreover, JNDs may change during the course of rehabilitation as a patient's sensory and motor capacities improve or otherwise change [6].

In the study we describe here we explore force JNDs in healthy subjects using a methodology that we will transfer to the study of the disabled. The resultant JNDs will ultimately be used as a tool to calibrate therapeutic distortions in virtual environments and as a means to create environments that adapt. Our consideration of 
JNDs, then, holds them to be dynamic properties that may change over time.

Moreover, the technique used here is responsive to the fact that people do not produce constant forces spontaneously unless they are artificially controlled (e.g., by a cliff or wall) [7,9]. Because artificial terminations will not be available when patients independently produce force during therapy, the current task has subjects gradually increase self-produced force to a peak value that is visually signaled.

Initially, we have asked subjects to sample two sets of simulated forces applied to the finger from a continuous range and to indicate whether or not the sets of forces feel the same. From the results we have gathered from healthy subjects, we have computed thresholds of sensitivity and force JNDs. These initial results will provide a baseline for future study.

\section{Methods}

Subjects for the study described were five healthy male members of the Carnegie Mellon community; all were right handed. No subject was known to have any neurological disorders.

Each subject was seated on a chair facing a computer monitor and asked to place his or her right forearm on a side support (see Figure 1). The index finger of the right hand was secured within a splinted attachment to a forcefeedback device called the PHANToM ${ }^{\mathrm{TM}}$. All joints of the finger save for the index metacarpo-phalangeal joint were restrained so that finger movements were locked into a semi-circular trajectory about the free joint. The forearm was also restrained, so as to minimize shifting during experimental runs.

Once a subject was seated comfortably and secured, his or her right arm and fingers were shielded from view with an opaque piece of black paper. Attention was directed to the monitor containing a graphical display. This display was rendered in Open GL using the Ghost $\mathrm{SDK}^{\mathrm{TM}}$ on a 4 GB $733 \mathrm{MHz}$ Pentium III PC running Windows NT 4.0. The machine had 260 MB of RAM.

During each run, subjects were asked to sample pairs of forces by pressing with their index finger against simulated virtual "springs". Each spring was simulated with the PHANToM ${ }^{\mathrm{TM}}$, and its force was delivered such that it lay tangential to the trajectory of the finger. Subjects pressed against these springs until their finger lay in a target window; a green box on the graphical display indicated when the finger was within the bounds of a target force window (as per Figure 1). The graphical box above the central one (the target) was highlighted if the finger was before its target, and the box below was highlighted if the finger moved too far. Subjects were required to maintain the finger within the target window for two seconds, at the end of which time they were assumed to have adequately sampled the target force.

The desired force and spring constant determined the angle along the finger arc where the target force occurred. By varying the spring constant from trial to trial, terminal finger positions corresponding to a given target force changed location. This variation was designed to discourage individuals from judging force based on finger position or the damping constant of the spring. Moreover, the variation insured that resulting force JNDs were independent of other variables.

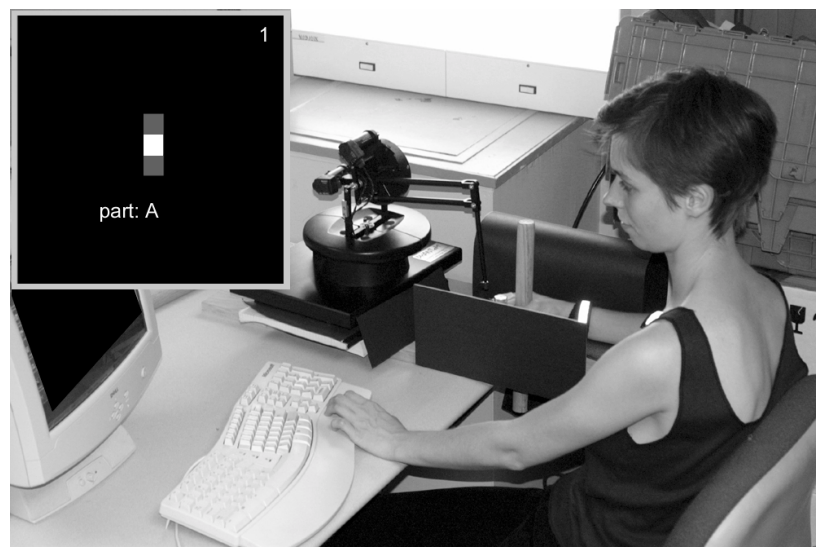

Figure 1. The experimental setup. Subjects sampled pairs of forces with their right index finger by pressing against virtual "springs" simulated by the PHANToM ${ }^{\mathrm{TM}}$ device. The image on the computer display (here, in the top left) indicated when the subject had pressed against the springs to a target position. During each run, subjects' hands were shielded from view with an opaque sheet of black paper.

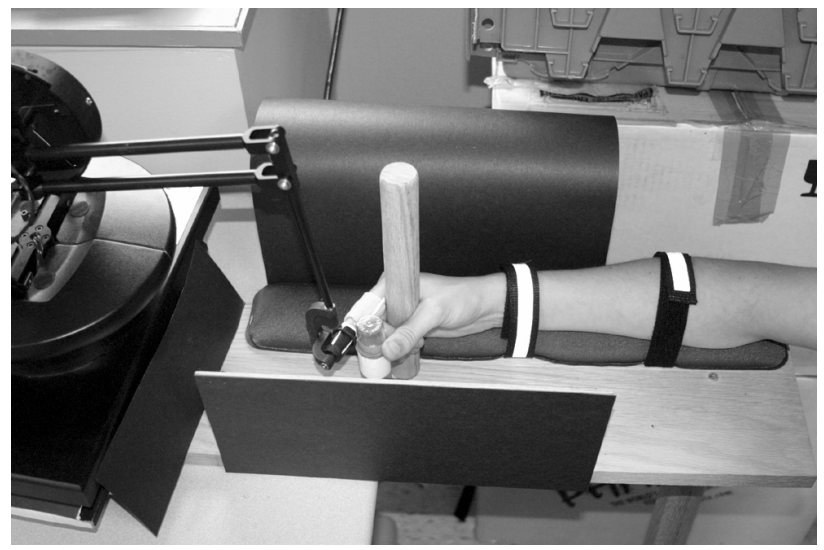

Figure 2. The experimental setup, top view. All joints of the finger, save for the index metacarpo-phalangeal joint, were restrained. Forces were issued to the index finger so that they lay tangential to the finger's semicircular trajectory. The forearm was also restrained, so as to minimize shifting during experimental runs. 
Each trial used the same graphical display; boxes on the screen display were purely iconic and did not reveal direct positional information. The range of spring constants that were experienced ranged between 50 and $100 \mathrm{~N} / \mathrm{m}$, and each motion required flexion of the finger along an arc length ranging between 30 and $40 \mathrm{mms}$.

Pairings of forces constituted two different stimulus conditions that were delivered with equal a priori probability. The first condition was a base force followed by the same base force and the second condition was a base force followed by an incremented force. In either case, use of the base force as the first member of the pair means that subjects re-sampled the referent force at the opening of each trial. After sampling a given force pairing, subjects were asked to judge the condition to which it belonged. They pressed an appropriate keyboard button with the opposite hand to indicate their judgment and feedback regarding accuracy was supplied after each trial. For the purpose of training, all subjects made at least ten comparisons before experimental trials began.

Each run consisted of 60 comparisons and each subject participated in one run. The base force was held at a constant 2.25 Newtons and the force increment was $20 \%$ of this base. This increment was set so that subjects achieved an accuracy level above chance but still made errors. The window around the target force -- base or augmented - allowed for diversion from the target that was bounded by $\pm 1 \%$. As such, 'sameness' between two base/base samplings may have in fact corresponded to a limited difference.

\section{Analysis of Results}

From the pattern of subjects' performance over a run, a sensitivity index (d') was computed for each given base force and increment. Technically:

$$
d^{\prime}=f^{-1}(a)-f^{-1}(b)
$$

where $\mathrm{a}$ is the hit rate, $\mathrm{b}$ the false alarm rate, and:

$$
f(x)=\frac{1}{\sqrt{2 \pi}} e^{\frac{-x}{2}}
$$

This d' assumes that a subject's perceptual response to a given stimulus condition can be modeled with a standard normal curve. Discrimination between two distinct stimulus conditions, then, involves discriminating between two overlapping perceptual response curves. The subject is assumed to adopt a decision criterion at a particular perceptual level, such that perceptual values above and below criterion are assigned to two different responses. This criterion value indicates the subject's bias to use one response more than another.

To indicate the criterion, we report $\beta$ as the ratio of the height of two normal curves at the perceptual level where the criterion is set. This $\beta$ is defined as:

$$
\beta=e^{z}
$$

where:

$$
z=\frac{1}{2}\left(f^{-1}(b)^{2}-f^{-1}(a)^{2}\right)
$$

A $\beta$ of 1.0 is unbiased, because it occurs where the two normal curves cross.

JNDs were computed as the percentage increment in force that led to a d' of 1 , which is occurs at $75 \%$ correct discrimination (assuming $\beta=1$ ). For the purpose of this study, we have defined the JND in terms of the $\delta$ statistic:

$$
\delta=\frac{d^{\prime}}{F}
$$

Here, $\mathrm{F}$ is the force increment over which a given d' was measured. JNDs have been computed as:

$$
\frac{1}{\delta} \times 100
$$

This reciprocal has then been scaled by a factor of 100 , so that the JND corresponds to a percentage.

\section{Results}

Figure 3 describes the resulting JNDs; one JND was computed for each subject. The results point to a JND of 9.9\%. Computed d's averaged 2.4 and the variance was bounded by $.15 \%$. As a group, they are very consistent, indicating similar task difficulty across subjects. Finally, for all results reported, subjects' $\beta$ values averaged 1.23, indicating that there was relatively little tendency to be biased in reporting.

\section{Discussion}

Our experimentally determined JND of roughly $10 \%$ is comparable to those JNDs derived from active weight lifting and force matching about the elbow. A number of significant elements, however, distinguish our paradigm from previous tasks.

The most significant of these differences is that our JNDs reflect judgments regarding a force window rather than a constant force; these windows allowed forces to 
deviate from the target by $1 \%$. We have included this attribute to facilitate further research that makes use of a force-matching framework, in which subjects sample a reference force and make an independent force production in an effort to match. On top of this framework, we will construct visual feedback distortions and thereby test the degree to which we can encourage augmentations in an individual's force production without awareness that augmentation is taking place. Information yielded through this paradigm will be of direct relevance to the construction of therapeutic virtual environments tailored to the domain of stroke rehabilitation.

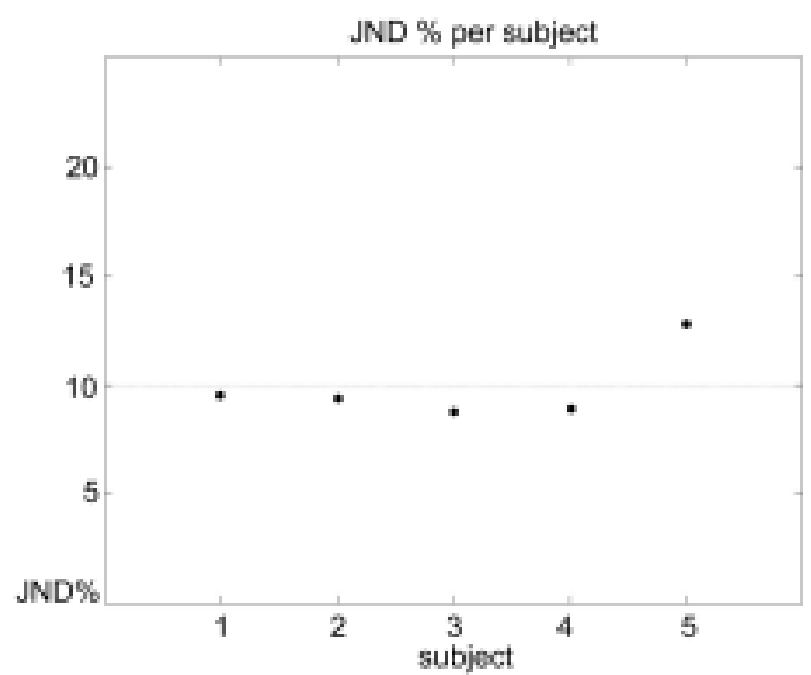

Figure 3. JNDs per subject. The dashed line lies at the average JND.

A $10 \%$ force JND, in fact, suggests that we may have a great deal of room in which to encourage such incremental augmentations of force production. Our results tell us that we should, for example, be able to ask for a $2 \mathrm{~N}$ production of force while recording a $2.2 \mathrm{~N}$ production, without the difference being reliably detected. Should a patient be resistant to the idea of extending his or her comfortable capacities in therapy, then, we can use such feedback distortions to subvert this resistance. Visual feedback can be designed to reinforce a patient's perception of ability, even if that perception is unreasonably limited. A therapist or device can structure true force exerted so as to reflect a patient's legitimate capacity.

In the immediate future, we will be exploring JNDs with respect to position and velocity, so that we may understand what kind of discrepant, yet imperceptible, feedback arrangements each type of JND will allow. We will once again be looking at these statistics in healthy individuals, and will be relating our results to JNDs in the stroke population; these data will be recorded in the coming months.

Our work thus far, however, provides a reasonable testbed upon which to build an understanding of sensory deficit in a disabled population. Moreover, the procedures we devise here can help us explore JNDs as they change during the course of recovery. Once this has been done, the development of adaptive rehabilitative environments tailored to patients' particular sensitivities can begin.

\section{Acknowledgements}

The first author is funded, in part, by an NSF Graduate Research Fellowship.

\section{References}

[1] Brodie, E. \& Ross, H. "Sensorimotor mechanisms in weight discrimination." Perception and Psychophysics, 36, 1984, pp. 477-481.

[2] Clark, F.J. and Horch, K.W. Kinesthesia. In Handbook of Perception and Human Performance v1.: Sensory Processes and Perception. (KR Boff, L Kaufman \& JP Thomas, eds.) New York: Wiley, 1986. pp. 13-1 - 13-62.

[3] Jones, L. A. "Matching forces: Constant errors and differential thresholds." Perception 18:5, 1989, pp.681-687.

[4] Jones, L. A. "Perception of Force and Weight: Theory and Research", Psychological Bulletin 100:1, 1986, pp. 29-42.

[5] Jones, L. A. "Visual feedback in the control of force", Exp Brain Res 130, 2000, pp. 269-272.

[6] Liepert, J., Bauder, H., Miltner, W.H.R., Taub, E., and Weiller, C. "Treatment-induced massive cortical reorganization after stroke in humans." Stroke, 31, 2000, pp. 1210 - 1216.

[7] Pang, X., Tan, H.Z. and Durlach,, N. "Manual discrimination of force using active finger motion," Perception \& Psychophysics 49: 6, 1991, pp. 531-540.

[8] Ross, H.E. and Murray, D.J. E.H. Weber: the sense of touch. New York: Academic Press, 1978.

[9] Srinivasan, M.A. and Chen, J.S., "Human performance in controlling normal forces of contact with rigid objects",

Advances in Robotics, Mechatronics, and Haptic Interfaces 49, ASME, 1993.

[10] Taub, E. Overcoming learned nonuse: A new behavioral medicine approach to physical medicine. In Clinical Applied Psychophysiology (J. G. Carlson, S. R. Seifert, \& N.

Birbaumer, eds.) New York: Plenum, 1994, pp. 185 - 220. 